\title{
Situação da malária na Região do Baixo Amazonas, Estado do Pará, Brasil, de 2009 a 2013: um enfoque epidemiológico*
}

Malaria situation in the Lower Amazon Region, Pará State, Brazil, from 2009 to 2013: an epidemiological approach

Situación de la malaria en la Región del Bajo Amazonas, Estado de Pará, Brasil, de 2009 a 2013 : un enfoque epidemiológico

Jonata Ribeiro de Sousa

Universidade do Estado do Pará, Núcleo Tapajós, Santarém, Pará, Brasil

Anne Caroline Farias dos Santos

Faculdades Integradas do Tapajós, Santarém, Pará, Brasil

Wagner de Sousa Almeida

Universidade do Estado do Pará, Núcleo Tapajós, Santarém, Pará, Brasil

Kaio Vinícius Paiva Albarado

Universidade do Estado do Pará, Núcleo Tapajós, Santarém, Pará, Brasil
Lizangela Dias Magno

Universidade do Estado do Pará, Santarém, Pará, Brasil

José Almir Moraes da Rocha

Departamento de Morfologia e Ciências Fisiológicas, Universidade do Estado do Pará, Núcleo Tapajós, Santarém, Pará, Brasil

Zilma Nazaré de Souza Pimentel

Departamento de Saúde Integrada, Universidade do Estado do Pará, Núcleo Tapajós, Santarém, Pará, Brasil

\section{RESUMO}

O objetivo da pesquisa foi conhecer as características epidemiológicas da malária na Região do Baixo Amazonas, Estado do Pará, Brasil. Para isso, foi feito um levantamento estatístico retrospectivo e descritivo da ocorrência de agravos à saúde por espécies de plasmódios causadores da malária na Região do Baixo Amazonas, no período de janeiro de 2009 a dezembro de 2013. Os resultados apontam a presença constante da malária nos municípios da região, sendo notificados 16.765 casos no período do estudo. Os Municípios com maior número de casos foram Oriximiná, Santarém, Alenquer e Prainha. Houve prevalência das infecções no sexo masculino. Observou-se maior incidência nas faixas de 20 a 29 anos de idade, com 20,51\% e 30 a 39 anos, com 14,96\%. A faixa etária de 5 a 9 anos apresentou $12,47 \%$ dos casos. O Plasmodium vivax foi mais prevalente $(76,84 \%$ de todos os casos) em todos os municípios da região. A maioria dos municípios foi classificada como áreas de baixo risco de transmissão da malária; houve predomínio das formas de detecção passiva da doença e $75,1 \%$ dos casos foram classificados como autóctones. Houve registro de casos em gestantes e, estes, só ocorreram nos anos de 2009, 2010 e 2011 . É marcante a presença da malária na região, configurando-se em um grande desafio de ordem social, política e econômica, sobretudo por suas fortes repercussões na saúde da população. Sendo assim, as ações de controle devem ser mantidas e envolver, necessariamente, todos os segmentos governamentais.

Palavras-chave: Inquéritos Epidemiológicos; Malária; Saúde Pública.

\footnotetext{
* Os resultados parciais desta pesquisa foram publicados no Livro de Resumos da Feira de Trabalhos Acadêmicos e Científicos - FETAC, 2014 , Ano IV, Vol. 01 (2014), 118 p. ISSN 2238-806X.
}

\author{
Jonata Ribeiro de Sousa \\ Av. Marechal Rondon, 2963. Bairro: Aparecida \\ CEP: 68040-070 Santarém-Pará-Brasil \\ Tel.: +55 (93) 99213-6941 \\ E-mail: jsousastm@hotmail.com
}

Correspondência / Correspondence / Correspondencia: 


\section{INTRODUÇÃO}

A malária mostra forte influência sobre a saúde humana desde os tempos de outrora e, apesar de antiga, ainda representa grave problema de saúde pública, principalmente em áreas tropicais e subtropicais'. Afeta 198 milhões de pessoas no mundo e causa 584 mil mortes (variando de 367.000-755.000 mortes), principalmente na América, Ásia e África²

Cerca de 3,3 bilhões de pessoas $140 \%$ da população mundial) estão sob risco de contrair a doença, sendo que 1,2 bilhões de pessoas estão sob alto risco (a probabilidade de contrair a doença é >1/1.000). A mortalidade é bastante expressiva, principalmente em crianças menores de 5 anos de idade (78\% das mortes) e em mulheres grávidas ${ }^{2}$.

Países com nível de pobreza acentuado, com altos níveis de exclusão social, localizados, principalmente na África, são responsáveis por mais de $90 \%$ de todos os casos. Sendo assim, a malária é considerada o maior problema de saúde pública em muitos países, principalmente naqueles em desenvolvimento ${ }^{2}$.

No Brasil, fatores socioeconômicos e ambientais, como a migração interna, grandes projetos agropecuários, construção de rodovias e hidrelétricas, garimpo e mineração, têm sido determinantes na dinâmica da transmissão da malária, principalmente na Região Amazônica. Esses fatores favorecem a proliferação do vetor da doença e, consequentemente, a exposição de grandes contingentes populacionais ${ }^{3}$.

No período de 2000 a 2011 foram notificados 5.074.292 casos de malária, destes, 99,7\% ocorreram na Região Amazônica, considerada área endêmica no País. Nesses 12 anos, foi notificada uma média de 422.858 casos $^{4}$.

Estado do Pará reflete essa realidade regional. Parente et $a^{5}$ apontaram que o Pará vinha demonstrando tendência crescente no número de casos de malária, chegando a ocupar o topo da lista dos Estados que mais notificam a doença durante os anos de 2010 e 2011.

amplo espectro de distribuição, a importância epidemiológica, o potencial de gravidade clínica, justificada pelos altos índices da doença na Região Amazônia e no Estado do Pará endossam a realização deste estudo.

Nesse sentido, conhecer a dinâmica epidemiológica da malária na Região do Baixo Amazonas é crucial para dimensionar e problematizar a temática junto às esferas de saúde competentes, subsidiando a formulação de estratégias/políticas efetivas no controle da malária e fortalecimento do sistema de vigilância epidemiológica.

\section{MATERIAIS E MÉTODOS}

Foi realizado um levantamento estatístico, retrospectivo e descritivo dos casos de malária notificados na Região do Baixo Amazonas, no período de janeiro de 2009 a dezembro de 2013.
A Região do Baixo Amazonas é formada pelos Municípios de Alenquer, Almerim, Belterra, Curuá, Faro, Juruti, Monte Alegre, Óbidos, Oriximiná, Prainha, Santarém, Terra Santa; e ocupa 25,32\% do território do Estado. A região possuía 659.321 habitantes em 2008, correspondendo a $8,87 \%$ da população total do Estado do Paráb.

Quatro municípios da região (Belterra, Curuá, Faro e Terra Santa) são de pequeno porte, com população inferior a 20 mil habitantes; Almeirim, Juruti, Óbidos e Prainha são de porte intermediário a pequeno (população entre 20 mil a 50 mil). Os Municípios de Alenquer, Monte Alegre e Oriximiná, de porte intermediário, têm contingente populacional entre 50 mil a 100 mil habitantes. Já o Município de Santarém corresponde a 41,96\% (276.665 habitantes) da população do Baixo Amazonas, sendo, portanto, o polo regional da região ${ }^{6}$.

As variáveis abordadas neste estudo incluem: número de casos por ano, município de ocorrência, faixa etária, sexo, agente etiológico envolvido (espécie de plasmódio), tipo de detecção dos casos, casos autóctones e alóctones, índice parasitário anual (IPA) e infecção durante a gravidez.

O IPA estima o risco de ocorrência de malária numa determinada população em uma região circunscrita. As áreas são classificadas como de alto, médio e baixo risco de transmissão (maior que 49,9/1.000 habitantes: alto risco; entre 10 e 49,9/1.000 habitantes: médio risco; de 0,1 a 9,9/1.000 habitantes: baixo risco e IPA igual a zero: áreas sem risco de transmissão de malária) ${ }^{7}$; calcula-se a partir da seguinte equação:

$$
\mathrm{IPA}=\frac{\text { NEP. }}{P} 1000
$$

Onde:

NEP: número de exames positivos no período

\section{$P$ : população no período}

Os dados foram extraídos/coletados por meio do acesso ao banco de dados do Sistema de Vigilância Epidemiológica da Malária (SIVEP-MALÁRIA) da Secretaria de Vigilância em Saúde do Ministério da Saúde, após autorização por escrito da Divisão Técnica (DT) da $9^{a}$ Regional de Saúde (SESPA), com sede no Município de Santarém, e pela direção do setor local de endemias.

O SIVEP-MALÁRIA é o principal banco de dados de malária no Brasil. Está em vigor desde 2003 e funciona por meio da inserção, em um banco virtual, de dados inerentes às fichas de notificação compulsória da doença, veiculados pela Internet, o que permite maior vigilância pelos órgãos municipais, estaduais e federais ${ }^{8}$.

Considerou-se, para o estudo, todos os casos novos de malária notificados no período de janeiro de 2009 a dezembro de 2013 na Região do Baixo Amazonas que estivessem disponíveis por meio de acesso ao SIVEP-MALÁRIA; foram excluídos os exames laboratoriais classificados como lâminas de verificação de cura (LVC), pois estariam representando duplicidade de caso. Os dados foram analisados pelo programa Microsoft Excel versão 2010 e organizados em forma de tabelas e gráficos. 


\section{RESULTADOS}

Durante o intervalo amostral do estudo, foram notificados 16.765 casos de malária, sendo que, destes, 10.781 (64,3\%) ocorreram em indivíduos do gênero masculino, com predomínio em todos os anos. A tabela 1 mostra o panorama geral da distribuição da malária, de acordo com o município de notificação e IPA ao longo dos anos estudados. Nesta, confirma-se - caráter endêmico da malária, constatando a sua presença em todos os municípios da região, nos anos de 2009, 2010, 201 1, 2012 e 2013, à exceção de Faro e Terra Santa que não registraram casos em 2012 e 2013.

A maioria dos municípios foi classificada como área de baixo risco de transmissão de malária, sendo que em 2013, nove de 12 municípios da região (Belterra, Curuá, Faro, Juruti, Monte Alegre, Óbidos, Oriximiná, Prainha e Terra Santa) corroboraram este perfil; o IPA de médio risco esteve presente em 2009, 2010 e 2011 , nos Municípios de Alenquer e Prainha, mas com tendência decrescente nos anos consecutivos. Não houveram áreas sem risco de transmissão.

Houve maior número de casos nas faixas etárias de 20 a 29 e 30 a 39 anos, com percentual conjunto de $35,47 \%$ dos casos. As faixas etárias correspondentes à infância e adolescência também apresentaram elevado número de casos $(12,47 \%, 11,93 \%, 10,97 \%, 5$ a 9, 10 a 14 e 1 a 4 anos de idade respectivamente); 2,19\% ocorreram em crianças menores de 1 ano de idade (Figura 1).

Tabela 1 - Distribuição dos casos de malária, por municípios da Região do Baixo Amazonas, no período de janeiro de 2009 a dezembro de 2013

\begin{tabular}{|c|c|c|c|c|c|c|c|c|c|c|c|c|c|c|c|c|}
\hline \multirow{2}{*}{$\begin{array}{l}\text { Município da } \\
\text { Região do Baixo } \\
\text { Amazonas }\end{array}$} & \multirow{2}{*}{ População } & \multicolumn{3}{|c|}{2009} & \multicolumn{3}{|c|}{2010} & \multicolumn{3}{|c|}{2011} & \multicolumn{3}{|c|}{2012} & \multicolumn{3}{|c|}{2013} \\
\hline & & $\mathrm{N}$ & $\%$ & IPA & N & $\%$ & IPA & $\mathrm{N}$ & $\%$ & IPA & $\mathrm{N}$ & $\%$ & IPA & $\mathrm{N}$ & $\%$ & IPA \\
\hline Alenquer & 52.626 & 898 & 14,68 & 17,06 & 753 & 19,67 & 14,31 & 1.479 & 40,90 & 28,10 & 662 & 32,50 & 12,58 & 304 & 26,12 & 5,78 \\
\hline Almeirim & .614 & 296 & 4,84 & 8,81 & 257 & 6,71 & 7,65 & 343 & 9,49 & 10,20 & 179 & 8,79 & 5,33 & 245 & 21,05 & 7,29 \\
\hline Belterra & 16.318 & 35 & 0,57 & 2,14 & 14 & 0,37 & 0,86 & 22 & 0,61 & 1,35 & 43 & 2,11 & 2,64 & 10 & 0,86 & 0,61 \\
\hline Curuá & 12.254 & 12 & 0,20 & 0,98 & 4 & 0,10 & 0,33 & 44 & 1,22 & 3,59 & 7 & 0,34 & 0,57 & 4 & 0,34 & 0,33 \\
\hline Faro & .177 & 316 & 5,16 & 38,64 & 66 & 1,72 & 8,07 & 56 & 1,55 & 6,85 & - & - & 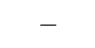 & - & - & - \\
\hline Juruti & 47.086 & 8 & 0,13 & 0,17 & 14 & 0,37 & 0,30 & 1 & 0,03 & 0,02 & 5 & 0,25 & 0,11 & 13 & 1,12 & 0,28 \\
\hline Monte Alegre & 55.462 & 49 & 1,02 & 1,19 & 169 & 1 & 3,05 & 31 & 4 & 1,40 & 52 & 2,55 & 4 & 50 & 4,30 & 0,90 \\
\hline Óbidos & 49.333 & 147 & 2,40 & 2,98 & 102 & 2,66 & 2,07 & 201 & 5,56 & 4,07 & 78 & 3,83 & 1,58 & 29 & 2,49 & 0,59 \\
\hline Oriximiná & 62.794 & 2.672 & 43,67 & 42,55 & 500 & 13,06 & 7,96 & 80 & 2,21 & 1,27 & 21 & 1,03 & 0,33 & 43 & 3,69 & 0,68 \\
\hline Prainha & .047 & 626 & 10,23 & 21,331 & 1.019 & 26,61 & 34,72 & 471 & 13,03 & 16,05 & 264 & 12,96 & 9,00 & 10 & 0,86 & 0,34 \\
\hline Santarém & 294.580 & 966 & 15,79 & 3,28 & 921 & 24,05 & 3,13 & 834 & 23,06 & 2,83 & 726 & 35,64 & 2,46 & 456 & 39,18 & 1,55 \\
\hline Terra Santa & 16.949 & 44 & 0,72 & 2,60 & 10 & 0,26 & 0,59 & 4 & 0,11 & 0,24 & - & - & - & - & - & - \\
\hline Total & 678.542 & 6.119 & 100,00 & 5,2 & 3.829 & 100,00 & 83,02 & 3.616 & 100,00 & 76,0 & 2.037 & 100,00 & 35,53 & 1.164 & 100,0 & 18,34 \\
\hline
\end{tabular}

Sinal convencional utilizado: - Dado numérico igual a zero não resultante de arredondamento.

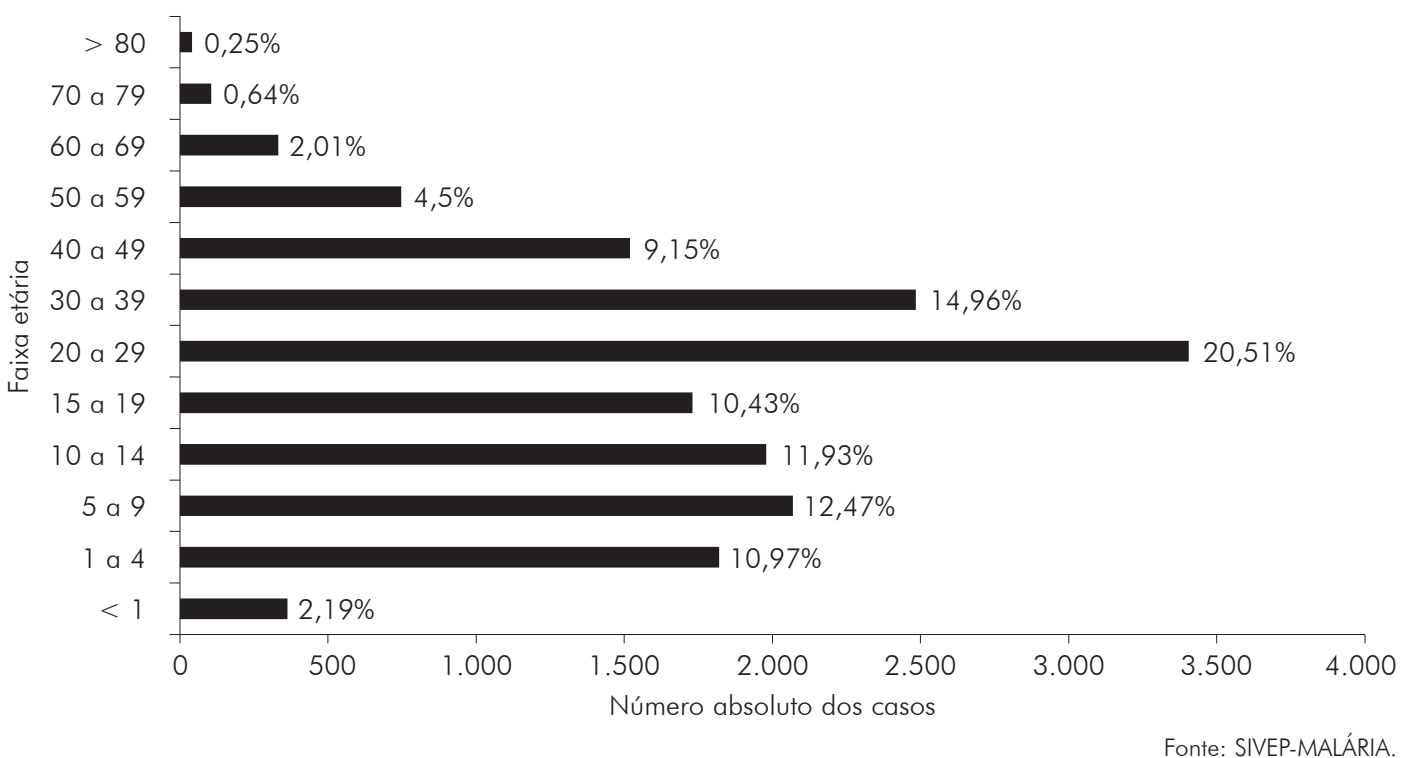

Figura 1 - Número de casos de malária, por faixa etária, na Região do Baixo Amazonas, no período de janeiro de 2009 a dezembro de 2013 
A infecção por Plasmodium vivax foi a mais notificada em todos os municípios, perfazendo um total de $76,8 \%$ de todos os casos notificados na região, com altas taxas de infecção em Almeirim (89,7\%), Belterra (95,2\%), Curuá (90,1\%), Prainha (98,7\%), Santarém (82,5\%) e Terra Santa (86,2\%). O Plasmodium falciparum $(21,5 \%$ do total de casos) esteve em evidência principalmente nos Municípios de Alenquer $(29,2 \%)$, Óbidos (49\%) e Oriximiná (36\%), considerando a proporcionalidade com a forma predominante (P. vivax). O Plasmodium malariae e a forma mista de infecção $(V+F)$ totalizaram $277(1,6 \%)$ casos, sendo que apenas cinco casos correspondem ao P. malariae e todos relativos ao Município de Almeirim (Figura 2).

Foi evidenciada maior prevalência, em todos os anos da pesquisa, das infecções por P. vivax. Em 2009, esta espécie foi responsável por 4.393 dos casos de malária, mantendo tendência decrescente ao longo dos anos (2010, 2011, 2012 e 2013, com 3.043, 2.741, 1.755 e 941 casos, respectivamente). As infecções por P. falciparum, também mostram pico mais alto no ano de 2009 (1.612 casos), decrescendo no ano de 2010 (756 casos), com leve aumento em 2011 (805 casos), com nova queda em 2012 (239 casos) e 2013 (192 casos). O P. malariae e a forma mista da malária (vivax + falciparum) também são evidenciados na pesquisa, na qual, juntos, perfazem cerca de 1,6\% do total de casos.

A variação anual das infecções por $P$. vivax nos municípios também mostra tendência decrescente, com exceção de Juruti (incremento de 61,5\% em 2013 em relação a 2009); os Municípios de Curuá, Faro, Óbidos, Oriximiná e Prainha, quando comparados ao número de casos de 2013 em relação a 2009, apresentam percentual de redução de 75\%, $100 \%, 90,2 \%, 97,5 \%$ e $98,7 \%$, respectivamente. 0 P. falciparum manteve padrão semelhante (decrescente), com exceção de Óbidos que apresentou inversão dos índices de infecção por P. falciparum em detrimento do P. vivax a partir de 2011 (9,5\%, 22,5\%, 87,1\%, 64,1\%, 55,2\% em 2009, 2010, 2011, 2012, 2013, respectivamente). Na análise comparativa (2009/2013), destaque para Oriximiná que reduziu para quatro casos em 2013 um quantitativo de 1.021 identificados em 2009; Faro, com nenhum caso em 2013, 110 em 2009; Almeirim, oito casos em 2013, 52 em 2009; Prainha, dois casos em 2013, 16 em 2009; Terra Santa com nenhum caso em 2013, sete em 2009.

Do total de casos de malária notificados no intervalo amostral, 9.087 (54,3\%) foram diagnosticados pela detecção passiva, que se mostrou predominante em todos os anos, exceto em 2011 , em que $51 \%$ dos casos foram diagnosticados por meio da busca ativa. Foram classificados $75,1 \%$ como autóctones, sendo que em 2009 estes representavam 84,2\%, em 2010, 77,8\%, em 2011 , 72,2\%, em 2012, 61,7\% e em 2013, 50,5\% dos casos.

A figura 3 delineia a procedência (se autóctone ou importados), o tipo de detecção e o município de notificação dos casos de malária, evidenciando predomínio das formas de detecção passiva sobre a ativa em nove municípios da região, sendo eles: Almeirim (61,5\%), Curuá (85,9\%), Faro (66\%), Juruti (97,6\%), Monte Alegre (72,4\%), Óbidos (58,3\%), Oriximiná (64\%), Santarém $(85,1 \%)$ e Terra Santa (94,8\%). Nos municípios de Alenquer (68,6\%), Belterra (68,5\%) e Prainha (70,3\%) houve maior percentual de detecção ativa. É possível verificar, também, que os Municípios de Alenquer (3.954 casos), Almeirim (1.028 casos), Oriximiná (3.236 casos) e Prainha (2.206 casos) despontam com os principais municípios com grande número de casos autóctones, com total agregado de $82,9 \%$ de todos os casos autóctones. Os casos importados tiveram maior percentual nos Municípios de Belterra $(50,8 \%)$, Curuá $(74,6 \%)$, Juruti $(75,6 \%)$, Monte Alegre $(59,4 \%)$, Santarém $(71,2 \%)$ e Terra Santa $(89,7 \%)$.

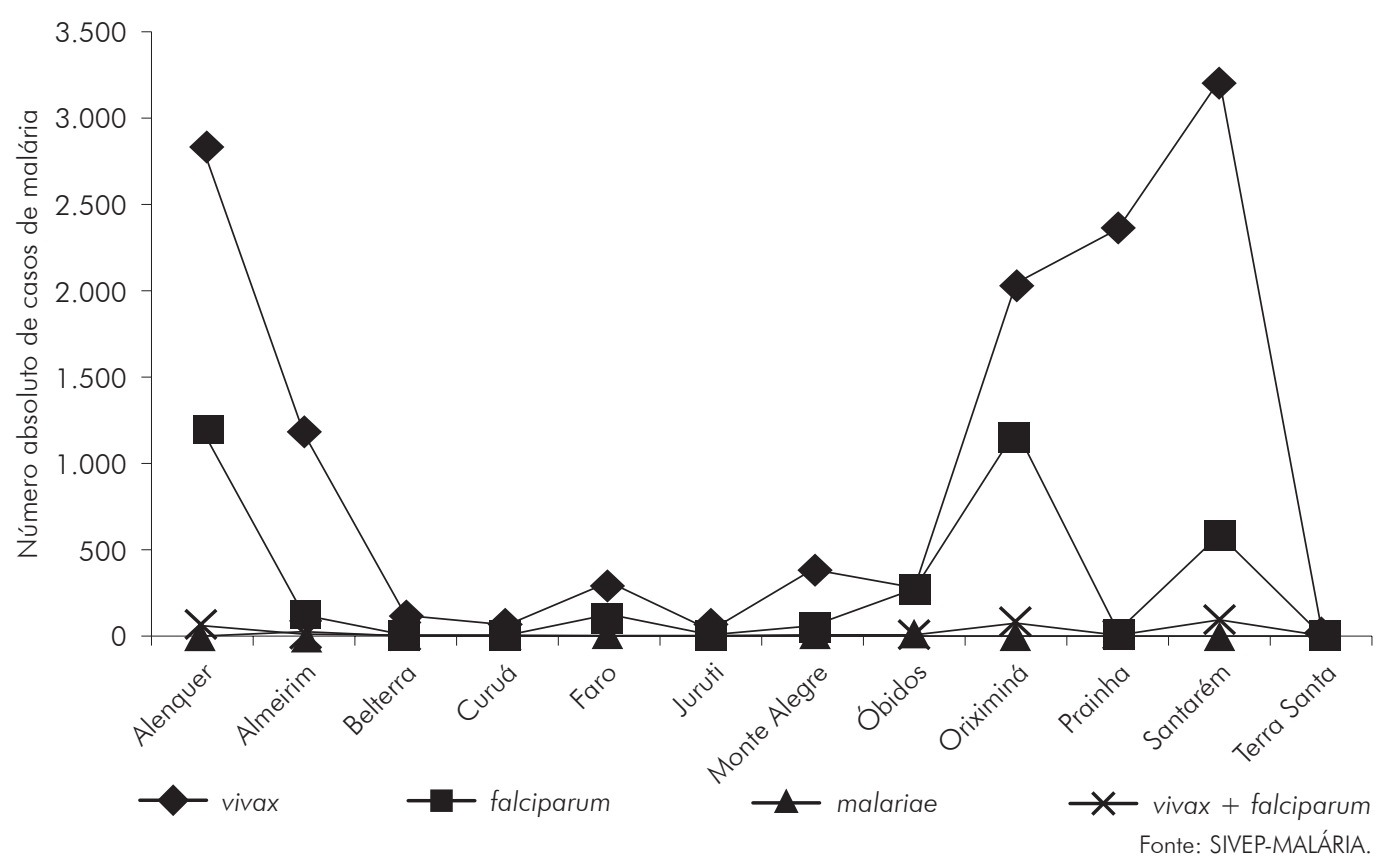

Figura 2 - Distribuição do número de casos acumulados de malária por município, segundo agente etiológico, na Região do Baixo Amazonas, no período de janeiro de 2009 a dezembro de 2013 
Dos 6.649 casos de malária em mulheres, 211 $(3,2 \%)$ ocorreram em grávidas. Só foram captados dados referentes as notificações nos anos de 2009, 2010 e 2011, pois houve mudança no sistema classificação desta variável (a partir de 2012), o que dificultou a extração destes dados no prazo autorizado para coleta de dados. A análise anual por município evidencia maior número de casos nos Municípios de Oriximiná, Prainha e Santarém, principalmente nos dois primeiros anos, conforme tabela 2. Em 2011, houveram cinco casos notificados, sendo um em Alenquer, um em Faro, um em Monte Alegre e dois em Santarém.

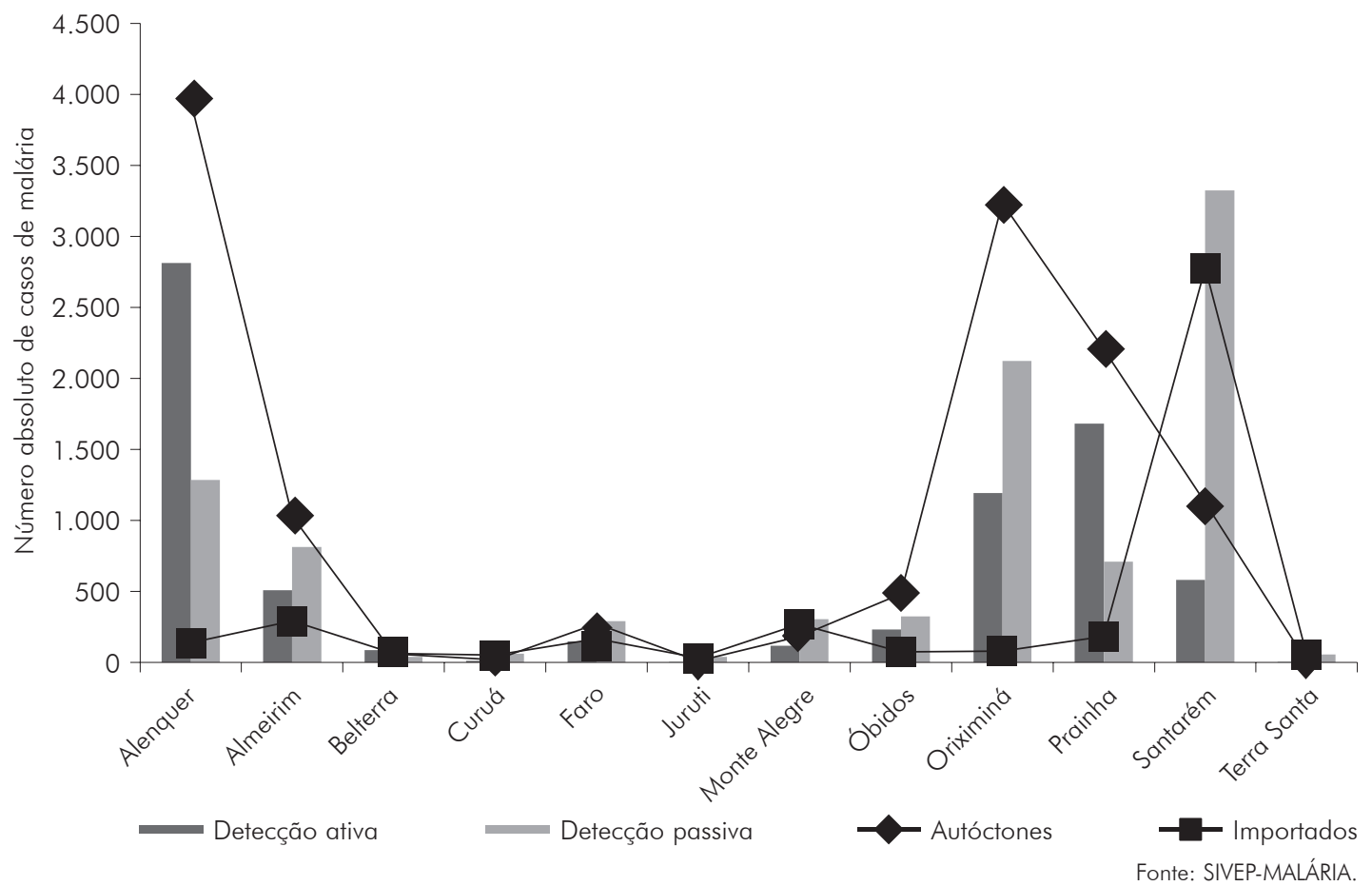

Figura 3 - Variação dos casos de malária, por município, segundo procedência e tipo de detecção, na Região do Baixo Amazonas, no período de janeiro de 2009 a dezembro de 2013

Tabela 2 - Casos de malária em gestantes, por município e ano de notificação, na Região do Baixo Amazonas, no período de janeiro de 2009 a dezembro de 2013

\begin{tabular}{|c|c|c|c|c|c|c|c|c|c|c|}
\hline \multirow{2}{*}{$\begin{array}{l}\text { Municípios da Região do } \\
\text { Baixo Amazonas }\end{array}$} & \multicolumn{2}{|c|}{2009} & \multicolumn{2}{|c|}{2010} & \multicolumn{2}{|c|}{2011} & \multicolumn{2}{|c|}{2012} & \multicolumn{2}{|c|}{2013} \\
\hline & N & $\%$ & $N$ & $\%$ & $\mathrm{~N}$ & $\%$ & $\mathrm{~N}$ & $\%$ & N & $\%$ \\
\hline Alenquer & 9 & 6,5 & 11 & 16,2 & 1 & 20,0 & - & - & - & - \\
\hline Almeirim & 6 & 4,3 & 4 & 5,9 & - & - & - & - & - & - \\
\hline Belterra & - & - & - & - & - & - & - & - & - & - \\
\hline Curuá & - & - & - & - & - & - & - & - & - & - \\
\hline Faro & 10 & 7,2 & - & - & 1 & 20,0 & - & - & - & - \\
\hline Juruti & - & - & - & - & - & - & - & - & - & - \\
\hline Monte Alegre & 7 & 5,1 & 5 & 7,4 & 1 & 20,0 & - & - & - & - \\
\hline Óbidos & 1 & 0,7 & 1 & 1,5 & - & - & - & - & - & - \\
\hline Oriximiná & 70 & 50,7 & 16 & 23,5 & - & - & - & - & - & - \\
\hline Prainha & 12 & 8,7 & 14 & 20,6 & - & - & - & - & - & - \\
\hline Santarém & 21 & 15,2 & 17 & 25,0 & 2 & 40,0 & - & - & - & - \\
\hline Terra Santa & 2 & 1,4 & - & - & - & - & - & - & - & - \\
\hline Total & 138 & 100,0 & 68 & 100,0 & 5 & 100,0 & - & - & - & - \\
\hline
\end{tabular}


número de casos de malária na Região do Baixo Amazonas apresentou redução significante em escala temporal. Em 2009, eram 6.119 casos, seguindo-se de 3.829, 3.616, 2.037 e 1.164 em 2010, 2011, 2012 e 2013, respectivamente. Houve redução de $81 \%$ dos casos notificados, comparando-se as cifras de 2009 com as de 2013. O percentual de redução do número de casos, por município (de 2009 para 2013) mostra que houve decréscimo de 66,1\% em Alenquer, 17,2\% em Almeirim, 71,4\% em Belterra, 66,4\% em Curuá, $100 \%$ em Faro, 49,4\% em Monte Alegre, 80,3\% em Óbidos, 98,4\% em Oriximiná, 98,4\% em Prainha, $52,8 \%$ em Santarém e 100\% em Terra Santa; houve incremento de $62,5 \%$ em Juruti.

\section{DISCUSSÃO}

No Estado do Pará, a presença da malária é marcante e se justifica pelas idiossincrasias geográficas, ecológicas, biológicas e socioeconômicas, que predispõem a transmissão da doença' ${ }^{9}$. Soma-se a isso, a herança histórica deixada pela política de ocupação da Região Amazônica, dirigida pelo Instituto Nacional de Colonização e Reforma Agrária (INCRA) entre as décadas de 1970 e 1980, que ocasionou intenso processo migratório para o interior do Estado e, favorecendo o crescimento populacional desordenado ao longo dos rios e das rodovias 9 .

A dificuldade de acesso aos bens e serviços necessários à promoção da saúde e melhoria das condições de vida tem favorecido a manutenção de epidemias em diversas localidades do Estado. Além disso, a falta de infraestrutura para circulação nessas áreas contribui para $o$ isolamento local e tem se tornado um obstáculo para o controle da endemia malarígena $^{10}$.

A diversidade dos cenários de transmissão da malária na Região Amazônica tem despertado a curiosidade e preocupação por parte de pesquisadores e órgãos governamentais relacionados, pois a doença destaca-se por seu aspecto "globocal" (termo de raiz sociológica, usado para se referir a problemas globais que apresentam idiossincrasias locais) ${ }^{11}$.

Os dados relacionados ao gênero assemelham-se ao de outras pesquisas ${ }^{7,12,13}$, no qual o gênero mais afetado pela malária é o masculino. A presença e permanência em locais de intensa transmissão (contato com o vetor), justificadas pelo tipo de atividade desenvolvida (caça, pesca, extrativismo, etc.) têm levado indivíduos do sexo masculino a maior exposição à doença ${ }^{14}$.

No Estado do Pará, o IPA da malária é classificado dentro do estrato de médio risco. Os municípios paraenses que apresentaram alto risco (IPA $\geq 50$ ) e médio risco $(50>\mathrm{IPA} \geq 10)$ de transmissão de malária tendem a diminuir em relação aos municípios com baixo risco (IPA $<10)^{9}$. E os municípios com baixo risco (IPA < 10) têm aumentado em relação ao total de municípios com alto e médio risco. A maior oferta de serviços de saúde aos doentes, em municípios com dificuldade de acesso, pode ter contribuído para esse panorama e, consequentemente, ter reduzido o total de municípios em situação de risco ${ }^{15}$. Na Região do Baixo Amazonas, ao longo de todos os anos estudados, a maioria dos municípios registraram baixo IPA e houve uma redução do número de municípios classificados como de risco médio, diminuindo para um município (Alenquer) em 2012.

A malária apresenta ampla distribuição em todos os segmentos etários, atingindo os extremos da vida e, principalmente nas faixas etárias ativas, de média idade $^{13}$, as quais estão em destaque neste estudo. No entanto, Costa et $a^{11}$ e Maciel et al ${ }^{13}$ também encontraram grande acometimento em faixas etárias mais jovens, entre escolares e pré-escolares (crianças com menos de 14 anos de idade), características, também, evidenciadas nesta pesquisa (incidência de até $39,3 \%$ neste mesmo grupo).

A supremacia das infecções por $P$. vivax, observadas neste estudo, corrobora as estatísticas estaduais? onde, dentre as espécies parasitárias mais prevalentes no Estado, observa-se que 86,3\% das infecções por malária são causadas pelo $P$. vivax, aproximadamente $12,1 \%$ por $P$. falciparum, a forma mais grave da doença e, a forma mista, totalizando 1,6\% (mesmo percentual deste estudo).

Um estudo de Santos e Silva ${ }^{8}$, feito por meio de série histórica (2003-2010), mostrou que P. vivax é a espécie mais prevalente, alcançando $73,9 \%$ dos casos autóctones notificados em oito anos. Gener ${ }^{16}$ apontou que a maior transmissibilidade de $P$. vivax está relacionada ao comportamento deste agente no hospedeiro vertebrado (homem), sobretudo no que tange ao tempo de formação dos gametócitos (formas infectantes para o mosquito), que é de $24 \mathrm{~h}$ para o $P$. vivax e de sete a 12 dias para o P. falciparum.

Apesar do predomínio atual da espécie P. vivax, outrora, o domínio era de P. falciparum. No Pará, tal fato ocorreu até 1990, quando observou-se aumento crescente do percentual de casos de malária pelo P. vivax no período de 1991 a 1999 .

A partir do ano de 2005, a Região Amazônica apresentou decréscimo dos casos de malária por P. vivax, com exceção apenas dos Estados do Amapá e Maranhão, cujas notificações apresentaram incremento de 18,7\% e 2,3\% - em 2013 com relação à 2005 -, respectivamente. As infecções por $P$. falciparum também caíram a partir do mesmo período, chegando a 12,0\% em 2011. A redução expressiva das infecções por P. falciparum tem sido relacionada à mudança, desde 2006, no uso da droga de primeira escolha para essa espécie, com substituição do esquema terapêutico com quinina e doxiciclina para combinações com derivados da artemisinina - antimaláricos ${ }^{4}$. Tais resultados também se aplicam aos municípios da Região do Baixo Amazonas, no que concerne as duas espécies em questão, uma vez que houve redução expressiva do número de casos totais. Fato curioso ocorreu no Município de Óbidos, no qual houve inversão do predomínio vivax/falciparum a partir de 2011, o que merece investigação detalhada dos fatores que levaram a tal desfecho. 
Apesar dessa redução, Parente et $a^{5}$ afirmaram que é evidente a presença da malária na Região Amazônica, sobretudo as infecções por P. vivax. Além disso, a infecção assintomática causada pelo P. vivax tem grande importância na manutenção da endemia por ser fonte de gametócitos (formas infectantes para - mosquito transmissor), pois representa dificuldade diagnóstica durante as ações de controle da transmissão da malária na região. Nesse sentido, o movimento populacional de pessoas portadoras de gametócitos e outras não imunes, associadas à proliferação do vetor, se constituem em importantes fatores de risco para o aumento do número de casos de malária por esta espécie?.

método de diagnóstico da malária no território nacional é feito ativamente, com realização de testes em domicílio ou em áreas de risco, ou de forma passiva, no qual os testes são feitos por demanda espontânea ${ }^{4}$.

De acordo com o Boletim Epidemiológico sobre a malária no Brasil, amiúde na Região Amazônica ${ }^{4}$, no período de 2003 a 2011, observou-se aumento no percentual de exames realizados de forma ativa na região; por outro lado, houve predominância, em todos os anos, do diagnóstico feito de forma passiva.

As condições geográficas, climatológicas e ecológicas favoráveis à transmissão da malária, são responsáveis pelos casos autóctones em uma região. Nesse sentido, Gener ${ }^{16}$ apontou que é necessário um aprofundamento da análise epidemiológica para caracterizar tal fenômeno, segundo variáveis de pessoa, espaço e tempo, assim como dos aspectos ambientais (socioeconômico, geográfico e meteorológico). Este estudo apoia tais considerações no sentido da realização de pesquisas mais aprofundadas sobre a temática, respeitando as peculiaridades de cada município.

incremento dos casos importados, no Município de Santarém, pode estar relacionado às características de município polo de saúde. Santarém é referência em serviços de Saúde para o oeste do Pará, devido aos seus recursos médicos assistenciais e as suas vias de acesso, tanto rodoviárias como, e principalmente, hidroviárias ${ }^{17}$.

As principais complicações da malária na gravidez estão relacionadas à integridade do binômio mãe-feto. As alterações que decorrem da gravidez (principalmente as de cunho imunológico) fragilizam o organismo materno, tornando-o suscetível às formas mais graves da doença, sendo as mais frequentes o aborto e parto prematuro - principalmente em áreas de baixa a moderada transmissão ${ }^{18}$. Outras complicações como a malária cerebral, edema pulmonar, anemia grave, hipoglicemia, morte neonatal representam uma ameaça constante ao organismo materno ${ }^{19}$.

Costa et $a^{11}$, ao avaliarem a ocorrência de infecção de malária em mulheres, detectaram taxa de 2,6\% relacionados à pacientes grávidas, o que mostra certa similaridade com o presente estudo. cenário de queda do número de casos de malária é nacional, constatando-se redução de até 40\%, em 2011 , na incidência da doença no País ${ }^{4}$.

Consonante com o Boletim Epidemiológico sobre malária, houve redução do coeficiente de transmissão da doença nos Estados da Região Amazônica, desde o ano de 2000 a 2011, exceto no Estado do Acre, em que houve incremento de $4,8 \%$ do total de casos. No Estado do Maranhão, a taxa de redução foi notória, alcançando 95,5\%; já o Estado do Pará ficou em quarto lugar com 58,8\% ${ }^{4}$.

É provável que tal avanço tenha sido em decorrência das ações do Plano de Intensificações de Controle da Malária na Amazônia Legal e do Programa Nacional de Prevenção e Controle da Malária9, que incluem, entre outras ações: combate ao vetor, vigilância epidemiológica, diagnóstico oportuno, implantação estratégica de laboratórios, educação em saúde junto às populações afetadas e melhoria das condições de trabalho da equipe de combate à endemias ${ }^{8}$.

\section{CONCLUSÃO}

Confirma-se, neste estudo, o caráter de endemicidade da malária na Região do Baixo Amazonas, corroborando o perfil amazônico e estadual da transmissão da malária. Essa endemia apresenta altos índices de infecção em indivíduos do sexo masculino, o que pode estar relacionado às atividades laborais desenvolvidas, sendo que esta peculiaridade foi evidenciada em muitos estudos analisados.

A malária acomete, principalmente, indivíduos adultos do gênero masculino; entretanto as faixas etárias correspondentes à infância e adolescência, também apresentaram elevado número de casos (2,19\% ocorreram em crianças menores de 1 ano de idade), - que é preocupante se considerarmos o risco de apresentarem, com maior frequência, formas graves da doença - principalmente nas primo-infecções.

Os índices identificados no sexo feminino e nas crianças e adolescentes podem ser evidência da expansão do vetor infectado nas zonas periurbana e/ou peridomiciliar, e demonstra a necessidade de políticas públicas direcionadas, como a vigilância por meio de inquérito entomológico dessas áreas.

De forma geral, observa-se uma redução da transmissão da malária, principalmente as formas mais incidentes ( $P$. vivax e $P$. falciparum) compatível com - cenário nacional e paraense, em graus variáveis de decréscimo, principalmente a partir de 2005, evidenciado no Boletim Epidemiológico sobre a malária, publicado em 2013.

O IPA, na maioria dos municípios da Região do Baixo Amazonas, foi classificado como de baixo risco, mas a análise temporal revela classificação de médio risco em alguns municípios, mantendo tendência decrescente ao longo dos anos do estudo. Apesar das notificações, dos casos de malária, serem feitas em sua maioria por detecção passiva, deve-se considerar a discussão (das necessidades de ajustes neste ponto) 
a partir da experiência de cada município, refletidas na análise do presente estudo e outro que possam ser aventados.

Os casos autóctones representaram 75,1\% do total de casos notificados na região. Alguns autores sugerem que tal característica está relacionada a fatores ambientais e/ou comportamentais (atividade humana exercida no local); nesse sentido, suscitamos o desenvolvimento de pesquisa (incluindo tais variáveis a nível local) para caracterizar melhor o fenômeno.

A ocorrência de malária na gravidez deu-se em $3,2 \%$ dos casos notificados no sexo feminino ao longo da pesquisa, o que representa um grave problema à saúde pública, considerando as repercussões da doença sobre o binômio mãe-feto, apontando para a necessidade da investigação dos fatores de risco relacionados ao fenômeno, na Região do Baixo Amazonas. Tal conhecimento é fundamental para a efetivação e/ou a elaboração de políticas específicas de prevenção e controle da malária em mulheres grávidas. A ausência de registro de casos em 2012 e 2013 pode estar relacionado a possibilidade de erro de notificação nas diversas etapas que envolvem este procedimento.

Por fim, quando se avalia a variação dos casos de malária ao longo dos anos, nota-se um perfil decrescente que pode estar relacionado às ações desenvolvidas pelo Plano de Intensificações de Controle da Malária na Amazônia Legal e do Programa Nacional de Prevenção e Controle da Malária.

\title{
Malaria situation in the Lower Amazon Region, Pará State, Brazil, from 2009 to 2013 : an epidemiological approach
}

\begin{abstract}
The objective of the research was to understand the epidemiological features of malaria in the Lower Amazon Region, Pará State, Brazil. A retrospective and a statistical survey of the occurrence of health problems for Plasmodium species which cause malaria in the Lower Amazon Region were conducted from January 2009 to December 2013. The results show a constant presence of malaria in municipalities of that region, with 16,765 cases reported during the study period. The Municipalities with the highest number of cases were Oriximiná, Santarém, Alenquer and Prainha. The prevalence of infection was in males. There was a higher incidence from 20 to 29 years old, with $20.51 \%$ and from 30 to 39 years old with $14.96 \%$. The age group 5-9 years old presented $12.47 \%$ of cases. The Plasmodium vivax was the most prevalent (76.84\% of all cases) in all municipalities. The majority of municipalities were classified as areas of low risk of malaria transmission; the presence of detection forms of passive disease was found in $75.1 \%$ of cases and they were classified as autochthonous. Malaria cases were registered in pregnant women that only occurred in 2009, 2010 and 2011. It is notable the presence of malaria in the region, and it presents a great social, political and economic challenge, especially for its strong impacts on population health. Thus, the control measures should be maintained and involve necessarily all government segments.
\end{abstract}

Keywords: Health Surveys; Malaria; Public Health.

\section{Situación de la malaria en la Región del Bajo Amazonas, Estado de Pará, Brasil, de 2009 a 2013: un enfoque epidemiológico}

\section{RESUMEN}

El objetivo de la investigación fue de conocer las características epidemiológicas de la malaria en la Región del Bajo Amazonas, Estado de Pará, Brasil. Para eso, se realizó un listado estadístico retrospectivo y descriptivo de la ocurrencia de agravamientos de la salud por especies de plasmodios causadores de la malaria en la Región del Bajo Amazonas, en el período de enero de 2009 a diciembre de 2013. Los resultados señalan una presencia constante de malaria en los municipios de la región, habiendo sido notificados 16.765 casos en el período del estudio. Los Municipios con mayor número de casos fueron Oriximiná, Santarém, Alenquer y Prainha. Hubo prevalencia de las infecciones en el sexo masculino. Se observó una mayor incidencia entre los 20 y 29 años de edad, con $20,51 \%$ y 30 a 39 años, con 14,96\%. La franja etaria de 5 a 9 años presentó un 12,47\% de los casos. El Plasmodium vivax fue más prevalente $(76,84 \%$ de todos los casos) en todos los municipios de la región. La mayoría de los municipios se clasificó como áreas de bajo riesgo de transmisión de malaria; hubo predominio de las formas de detección pasiva de la enfermedad y $75,1 \%$ de los casos se clasificó como autóctonos. Hubo registro de casos en gestantes que sucedieron los años de 2009, 2010 y 2011. Es bastante marcada la presencia de malaria en la región, configurándose en un gran desafío de orden social, político y económico, sobre todo por sus fuertes repercusiones en la salud de la población. Siendo así, deben mantenerse las acciones de control e involucrar, necesariamente, a todos los segmentos gubernamentales.

Palabras clave: Encuestas Epidemiológicas; Malaria; Salud Pública. 


\section{REFERÊNCIAS}

1 Rodrigues EC, Lopes Neto D. Controle da malária em um município Amazônico. Rev Latino-Am Enfermagem. 2011 nov-dez;19(6):[9 telas].

2 World Health Organization. World malaria report 2014 [Internet]. Geneva: WHO; 2014 [cited 2015 May 12]. Available from: http://www.who.int/ malaria/publications/world_malaria_report_2014/ en/.

3 Couto AA, Calvosa VS, Lacerda R, Castro F, Rosa ES, Nascimento JM. Controle da transmissão da malária em área de garimpo no Estado do Amapá com participação da iniciativa privada. Cad Saude Publica. 2001 jul-ago; 17(4):897-907.

4 Ministério da Saúde (BR). Secretaria de Vigilância em Saúde. Situação da malária no Brasil, 2000 a 2011. Bol Epidemiol. 2013;44(1):1.

5 Parente AT, Sousa EB, Ribeiro JBM. Ocorrência de malária em quatro municípios do Estado do Pará, 1988 a 2005, e sua relação com o desmatamento. Acta Amaz. 2012 mar;42(1):41-8.

6 Secretaria de Estado de Integração Regional (Pará). Diretoria de Integração Territorial. Atlas de Integração Regional do Estado do Pará. Belém: SEIR; 2010.

7 Ministério da Saúde (BR). Secretaria de Vigilância em Saúde. Guia de vigilância epidemiológica. 7. ed. Brasília: Ministério da Saúde; 2010. Caderno 10, Malária; p. 31-54. (Série Normas e manuais técnicos).

8 Santos IG, Silva RSU. Malária autóctone no Município de Rio Branco, Estado do Acre, Brasil, no período de 2003 a 2010. Rev Pan-Amaz Saude. 2011 dez;2(4):31-7.

9 Oliveira-Filho AB, Martinelli JM. Casos notificados de malária no Estado do Pará, Amazônia Brasileira, de 1998 a 2006. Epidemiol Serv Saude. 2009 jul-set; 18(3):277-84.

10 Pinheiro MCN, Bacelar MDR, Almeida SS, Silveira LCL. Endemias e desenvolvimento da Amazônia. In: Mello AF. O futuro da Amazônia: dilemas, oportunidades e desafios no limiar do século XXI. Belém: UFPA; 2002. p. 131-49.
11 Costa KMM, Almeida WAF, Magalhães IB, Montoya R, Moura MS, Lacerda MVGL. Malária em Cruzeiro do Sul (Amazônia Ocidental brasileira): análise da série histórica de 1998 a 2008. Rev Panam Salud Publica. 2010 nov;28(5):353-60.

12 Ferreira MU. Lacunas na área de pesquisa e desafios para o controle da malária no Brasil. Cad Saude Publica. 2011 dez;27(12):2284-5.

13 Maciel GBML, Espinosa MM, Atanaka-Santos M. Epidemiologia da malária no município de Colniza, Estado de Mato Grosso, Brasil: estudo descritivo do período de 2003 a 2009. Epidemiol Serv Saude. 2013 jul-set;22(3):465-74.

14 Lopes NFSN, Tadei WP, Brito LMO, Bezerra JMT, Pinheiro VCS. Malária no maranhão: análise dos fatores relacionados com a transmissão no período de 2005 a 2009. Rev Pesq Saude. 2013 jan-abr; 14(1):40-4.

15 Parise EV, Araújo GC, Pinheiro RT. Análise espacial e determinação de áreas prioritárias para o controle da malária, no Estado no Tocantins, 2003-2008. Rev Soc Bras Med Trop. 2011 jan-fev; 44(1):63-9.

16 Gener MES. Característica epidemiológica da malária autóctone no estado do Tocantins, Brasil, 1998-2006 [dissertação]. Brasília (DF): Universidade de Brasília, Programa de Pós-graduação em Medicina Tropical; 2008.

17 Bacha HA. Identificação molecular das espécies de leishmânia em lesões cutâneas de pacientes atendidos no Centro de Controle de Zoonoses de Santarém, Pará [tese]. São Paulo (SP): Universidade de São Paulo, Faculdade de Medicina; 2009.

18 Almeida LB, Barbosa MGV, Martinez-Espinosa FE. Malária em mulheres de idade de 10 a 49 anos, segundo o SIVEP- Malária, Manaus, Amazonas, 2003-2006. Rev Soc Bras Med Trop. 2010 mai-jun;43(3):304-8.

19 Tobón-Castaño A, Solano MA, Sánchez LGA, Trujillo SB. Retardo no crescimento intrauterino, baixo peso ao nascer e prematuridade em recém-nascidos de grávidas com malária, na Colômbia. Rev Soc Bras Med Trop. 2011 mai-jun;44(3):364-70. 